\title{
LOG-PERIODIC CRITICAL AMPLITUDES: A PERTURBATIVE APPROACH
}

\author{
BERNARD DERRIDA AND GIAMBATTISTA GIACOMIN
}

\begin{abstract}
Log-periodic amplitudes appear in the critical behavior of a large class of systems, in particular when a discrete scale invariance is present. Here we show how to compute these critical amplitudes perturbatively when they originate from a renormalization map which is close to a monomial. In this case, the log-periodic amplitudes of the subdominant corrections to the leading critical behavior can also be calculated.
\end{abstract}

Dedicated to Herbert Spohn, our colleague, friend and source of inspiration, on the occasion of his $65^{\text {th }}$ birthday

\section{INTRODUCTION}

Power laws with non integer exponents are common in the study of scale invariant structures, in particular in the theory of critical phenomena at second order phase transitions. In some cases, when the scale invariance is discrete [16, 36, 14, 32, 29, 18, 2, 11, the amplitude of the powerlaw acquires a periodicity, often called log-periodic oscillations: see [35, 21] for reviews on the topic. These oscillatory amplitudes are usually more difficult to calculate [13, 12, 7, 26] than the critical exponents. When the scale invariance is associated to a renormalization group map, they can be related to some properties of the map, for example to the shape of its Julia set, see [13, 8].

The goal of the present paper is to show that these oscillatory amplitudes can be computed perturbatively when the unperturbed map is simple enough, in our case a monomial map. To be more precise let us consider the following question: take the map $x \mapsto F(x)$ with

$$
F(x)=F_{\epsilon}(x)=x^{p}(1+\epsilon G(x)),
$$

where $p=2,3, \ldots$ and the perturbation $G(x)$ is sufficiently regular and has a finite limit for $x \rightarrow \infty$ (we will actually need a condition on the decay of $G^{\prime}(x)$ too, and this will be pointed out later on, see (4.7)). If $x_{*}$ is the largest real fixed point of the map (so that $x_{*}=F\left(x_{*}\right)$ ), this fixed point is unstable, that is $F^{\prime}\left(x_{*}\right)>1$, for $\epsilon>0$ sufficiently small. We will actually just focus on $F(x)$ for $x \geq x_{*}$. If $F^{(n)}(x)$ denotes the $n$-th iterate of the map $F$, the sequence $\psi_{n}(x)$ defined by

$$
\psi_{n}(x)=\frac{\log F^{(n)}(x)}{p^{n}},
$$

has a limit for all $x \geq x_{*}$

$$
\psi_{\infty}(x)=\lim _{n \rightarrow \infty} \psi_{n}(x)
$$

Date: July 24, 2018. 
One directly sees that $\psi_{\infty}(x)>0$ for $x>x_{*}$, while $\psi_{\infty}\left(x_{*}\right)=0$. The existence of the limit directly follows from

$$
\left|\psi_{n+1}(x)-\psi_{n}(x)\right| \leq p^{-n-1} \log \left(1+\epsilon \sup _{x \geq x_{*}}|G(x)|\right) .
$$

It is straightforward to see that the limit satisfies the functional equation

$$
p \psi_{\infty}(x)=\psi_{\infty}(F(x)),
$$

which goes under the name of Böttcher equation in the iterated function literature [3, 30, and one expects and sometime can prove - see for example [5, 8] - that

$$
\psi_{\infty}(x) \stackrel{x \succsim x_{*}}{\sim}\left(x-x_{*}\right)^{\alpha} B\left(-\frac{\log \left(x-x_{*}\right)}{\log F^{\prime}\left(x_{*}\right)}\right),
$$

where the exponent $\alpha$ is usually non-integer and $B(\cdot)$ is a strictly positive periodic function of period one.The exponent $\alpha$ has the well known expression

$$
\alpha=\frac{\log p}{\log F^{\prime}\left(x_{*}\right)},
$$

while the oscillatory amplitude $B(y)$ is usually hard to determine. One goal of the present paper is to show how to compute this oscillatory amplitude perturbatively (related small $\epsilon$ expansions can be found in [9]). We will see in Section 3 that all the subdominant corrections to (1.6) can be calculated as well since they all can be expressed in terms of the periodic function $B(\cdot)$.

Remark 1.1. (1.6) is easily guessed: if one assumes that $\psi_{\infty}(x) \sim\left(x-x_{*}\right)^{\alpha} C\left(x-x_{*}\right)$ as $x \searrow x_{*}$, with $y \mapsto C(y)$ defined for $y \in\left(0, y_{0}\right)$ for an arbitrary choice of $y_{0}>0$, continuous and such that $0<C_{\min }<C(y)<C_{\max }$, one gets from (1.5) that

$$
\left(x-x_{*}\right)^{\alpha} C\left(x-x_{*}\right) \sim \frac{F^{\prime}\left(x_{*}\right)^{\alpha}}{p}\left(x-x_{*}\right)^{\alpha} C\left(F^{\prime}\left(x_{*}\right)\left(x-x_{*}\right)\right),
$$

and this implies the value (1.7) of $\alpha$ and the fact $C\left(F^{\prime}\left(x_{*}\right) y\right)=C(y)$ for every $y$, that is that $C(\cdot)$ is multiplicatively periodic of period $F^{\prime}\left(x_{*}\right)$. Equivalently, $C(y)=$ $B\left(-\log \left(x-x_{*}\right) / \log F^{\prime}\left(x_{*}\right)\right)$, with $B(\cdot)$ periodic of period one, as in (1.6).

Remark 1.2. In what follows we will often assume that

$$
G(1)=0,
$$

so that $x_{*}=1$ when $\epsilon$ is sufficiently small. There is no loss of generality in doing so because the general case can be reduced to $G(1)=0$ by the change of variable $x \mapsto x / x_{*}$ and $x_{*}$ can easily be computed perturbatively in $\epsilon$

$$
x_{*}=1-\epsilon \frac{G(1)}{p-1}+\epsilon^{2} \frac{p G(1)^{2}+2 G(1) G^{\prime}(1)}{2(p-1)^{2}}+\ldots
$$

In this paper we will also discuss the case where the map $F(x)$ has the form

$$
F(x)=F_{p}(x)=q x^{p}+(1-q) P(x),
$$

where $p$ is a large positive integer, $q \in(0,1)$ and $P(\cdot)$ is a polynomial such that $P(1)=1$. We aim at understanding what the oscillatory behavior becomes for large $p$ (when $P(\cdot)$ and $q$ are fixed). The assumptions guarantee that, for $p$ sufficiently large, 1 is the largest fixed 
point (and it is unstable). Maps in this class are for example $\left(x^{p}+1\right) / 2$ and $\left(x^{p}+2 x^{2}-x\right) / 3$. We will see that for large $p$ the oscillatory amplitude can be sharply estimated. In a nutshell, the reason why the map becomes amenable for large $p$ is that sharp estimates of the iterated map $F^{(n)}(x)$ are easy as long as $x$ is very close to $x_{*}$ - the linear regime - and when $x$ is large, because the map there is close to $q x^{p}$. The only difficult part of the iteration is in between these two regimes: we will see however that for $p$ large this intermediate regime essentially reduces to one iteration which one performs explicitly.

\section{TWO EXAMPLES}

Let us discuss here briefly two different examples where the above question is relevant.

2.1. The wetting problem on a hierarchical lattice. One of the developments in the theory of critical phenomena by the renormalization group approach was to study statistical mechanical models on hierarchical lattices [4, 28, 23, 15, 31]. These lattices are usually constructed by a recursive procedure which is at the origin of a discrete scale invariance and which allows one to write exact renormalization transformations.

Here we consider the so-called diamond lattice [28, 15] (in fact a generalization of it) which is constructed as follows. One starts with a lattice composed of a single bond between two sites $A$ and $B$. Then at every iteration step each bond of the previous step is replaced by $p \times b$ bonds, namely by $b$ parallel paths of $p$ bonds each. Therefore if, at step 0 , one starts with a single bond between $A$ and $B$, then, after $n$ iterations, there are $(p b)^{n}$ bonds in the lattice, $b^{\left(p^{n}-1\right) /(p-1)}$ directed paths from $A$ to $B$, and each of these directed paths has a length $L_{n}=p^{n}$.

One of the simplest critical phenomenon one may consider on such a hierarchical lattice is the wetting transition [11, 19, 20]: in the wetting problem, all the bonds along a single path (the special path) from $A$ to $B$ have an energy $-a$ whereas all the remaining bonds on the lattice have energy 0 . The energy $E_{W}$ of a path $W$ from $A$ to $B$ is simply the number of bonds it has in common with the special path. Equivalently this energy $E_{W}$ is the sum of the energies of the bonds visited by the path $W$. Then the partition function, at temperature $T$, is defined as

$$
Z(T)=\sum_{W} \exp \left(-\frac{E_{W}}{T}\right),
$$

where the sum is over all the $b^{\frac{p^{n}-1}{p-1}}$ paths $W$.

When the temperature varies, one observes a transition between a bound phase, where typical paths have a non zero fraction of their length in common with the special path, to a free phase where this fraction vanishes. At infinite temperature, all paths have weight 1 and the partition function counts simply the total number $Z_{n}(\infty)$ of paths

$$
Z_{n}(\infty)=b^{\frac{p^{n}-1}{p-1}}=b\left(Z_{n-1}(\infty)\right)^{p},
$$

while at finite temperature, the partition function satisfies the following recursion

$$
Z_{n}(T)=Z_{n-1}(T)^{p}+(b-1) Z_{n-1}(\infty)^{p} .
$$

If one introduces the ratio $x_{n}=Z_{n}(T) / Z_{n}(\infty)$, it follows that

$$
x_{n}=F\left(x_{n-1}\right) \quad \text { where } \quad F(x)=\frac{x^{p}+b-1}{b},
$$


with $x_{0}=\exp (a / T)$. At the $n$-th step the free energy $f_{n}$ per unit length is

$$
f_{n}\left(x_{0}\right):=\frac{\log Z_{n}(T)}{L_{n}}=\frac{\log F^{(n)}\left(x_{0}\right)}{p^{n}}+\frac{\log Z_{n}(\infty)}{p^{n}} .
$$

which implies for the large $n$ limit $f_{\infty}$ of $f_{n}$ (recall (1.2) and (1.3) $)$

$$
f_{\infty}\left(x_{0}\right)=\psi_{\infty}\left(x_{0}\right)+\frac{\log b}{p-1} .
$$

Note that, while from the construction of the lattice $b=2,3, \ldots$ is an integer, the iteration (2.4) makes sense for every $b>1$. In what follows we will consider this generalization (see Remark 2.3 for another probabilistic interpretation of $Z_{n}$ in this generalized context). Finding the critical behavior of the free energy when the temperature is close to the wetting transition temperature, i.e. when $x_{0}$ is close to the fixed point $x_{*}$ of the map $F$, is a problem of the same nature as the one posed in the introduction and the question we address here is to try to determine the periodic function $B(\cdot)$ in front of the power law singularity

$$
f_{\infty}(x)-\frac{\log b}{p-1} \beth_{\beth_{*}}\left(x-x_{*}\right)^{\alpha} B\left(-\frac{\log \left(x-x_{*}\right)}{\log F^{\prime}\left(x_{*}\right)}\right) .
$$

The map (2.4) can be reduced to the form (1.1) or (1.11) in the two following cases

- when $b$ is close to 1 or when $b$ is large, by making the change of variable $x=b^{\frac{1}{p-1}} X$, the map (2.4) becomes

$$
X \rightarrow X^{p}+b^{-\frac{1}{p-1}}-b^{-\frac{p}{p-1}},
$$

which is of the form (1.1).

- when $b$ is arbitrary and $p$ is large, because the application (2.4) coincides with (1.11), with the choice $q=1 / b$ and $P(\cdot) \equiv 1$.

Remark 2.1. It is worth pointing out that the partition function of the wetting model on more general hierarchical lattices is obtained by iterating a polynomial of the form $F(x)=q_{0}+q_{1} x+\cdots+q_{p} x^{p}$, with the $q_{j}$ 's probability weights. Of course $F(1)=1$ and it is easy to see that there is at most another positive root: recall that the unstable fixed point $x_{*}$ on which we focus is the largest positive root. We record here the well known formula (e.g. [24, 11, [5]) that actually holds for every $x \in(0, \infty)$

$$
\psi_{\infty}(x)=\frac{\log q_{p}}{p-1}+\log x+\sum_{i=0}^{\infty} p^{-(i+1)} Q\left(F^{(i)}(x)\right),
$$

with $Q(x):=\log \left(F(x) x^{-p} / q_{p}\right)$. It is rather straightforward to extract from this formula that $\psi_{\infty}(\cdot)$ is (real) analytic on $\left(x_{*}, \infty\right)$ (e.g. [8]): in statistical mechanics terms this says that $x_{*}$ is the only critical point (note that with the definition (1.2, 1.3) one has $\psi_{\infty}(x)=0$ for $\left.x \in\left(0, x_{*}\right]\right)$.

2.2. The Galton Watson process. The Galton process is a simple model for the size of the lineage of an individual [25]. The generations do not overlap and all the population is replaced at every generation.

One starts with a single individual at generation $g=0$. Then each individual living at generation $g$ has a probability $q_{k}$ of having $k$ offspring at the next generation $g+1$. 
Therefore if $N_{g}$ is the number of individuals at generation $g$ (so $N_{0}=1$ ), one has

$$
N_{g+1}=\sum_{i=1}^{N_{g}} k_{i}
$$

where the $k_{i}$ 's are $N_{g}$ independent random variables which take the value $k$ with a probability $q_{k}$. The size $N_{g}$ of the population at generation $g$ is therefore a random variable. The average number of offsprings at generation $g$ is (see (2.10) ) given by

$$
\left\langle N_{g}\right\rangle=\left(\sum_{k} k q_{k}\right)^{g}=\langle k\rangle^{g} .
$$

So the expected size of the population grows exponentially for $\langle k\rangle>1$. It is well known, see e.g. [25], that if $\langle k\rangle \leq 1$ then after sufficiently many generations the population goes extinct. We will actually restrict to the supercritical case $\langle k\rangle>1$ and in this context it is natural to define [24, 25] the generating function of the ratio $N_{g} /\left\langle N_{g}\right\rangle$

$$
H_{g}(\lambda):=\left\langle\exp \left(\lambda \frac{N_{g}}{\left\langle N_{g}\right\rangle}\right)\right\rangle
$$

One can see from (2.10) that

$$
H_{g}(\lambda)=\sum_{k}\left(H_{g-1}\left(\frac{\lambda}{\langle k\rangle}\right)\right)^{k} q_{k}=F\left(H_{g-1}\left(\frac{\lambda}{\langle k\rangle}\right)\right)=F^{(g)}\left(H_{0}\left(\frac{\lambda}{\langle k\rangle^{g}}\right)\right),
$$

where $F(x)=\sum_{k} q_{k} x^{k}$. Then $H_{\infty}(\lambda):=\lim _{g \rightarrow \infty} H_{g}(\lambda)$ satisfies

$$
H_{\infty}(\lambda)=F\left(H_{\infty}\left(\frac{\lambda}{\langle k\rangle}\right)\right)
$$

This functional equation is known under the name of Poincaré equation [30] and $H_{\infty}(\cdot)$ is the unique solution of (2.14) which is analytic in a neighborhood of the origin and such that $H_{\infty}(0)=1$ and $H_{\infty}^{\prime}(0)=1$ [30]. Note that that, given $H_{\infty}(0)$ and $H_{\infty}^{\prime}(0)$, one can extract recursively from (2.14) all the coefficients of the Taylor expansion at $\lambda=0$.

Remark 2.2. To be precise, the limit exists in great generality and it is (obviously) finite if $\lambda \leq 0$ [25, Ch. I]. In fact the random variable $W_{g}:=N_{g} /\left\langle N_{g}\right\rangle$ converges almost surely to a limit random variable $W_{\infty}$ as soon as $\langle k\rangle\langle\infty$ : this is particularly elementary if $\left\langle k^{2}\right\rangle<\infty$, because in this case one can explicitly compute $\left\langle\left(W_{g+n}-W_{g}\right)^{2}\right\rangle$ for $n \in \mathbb{N}[25$, p. 13] and establish the convergence (in probability) of $W_{g}$ to $W_{\infty}$ and the fact that $W_{\infty}$ is not identically zero. Of course in this generality $H_{\infty}(\lambda)$ can be $\infty$ for $\lambda>0$, but we will focus on the case in which only a finite number of $q_{k}$ 's are non zero and it is therefore rather straightforward to see that for every $\lambda>0$ there exists $C_{\lambda}$ such that

$$
\sup _{g}\left\langle\exp \left(\lambda W_{g}\right)\right\rangle \leq C_{\lambda} .
$$

This not only implies that $H_{\infty}(\lambda)$ is finite for every $\lambda \in \mathbb{R}$ and that it is equal to $\left\langle\exp \left(\lambda W_{\infty}\right)\right\rangle$, but also that the same is true for any $\lambda$ complex: as a matter of fact $H_{\infty}(\cdot)$ is an entire function, that is analytic on the whole of $\mathbb{C}$. 
Let us therefore assume that only a finite number of $q_{k}$ 's are non zero, so that the map $F(x)$ is polynomial of degree $p=\max \left\{k: q_{k} \neq 0\right\}$. T. E. Harris has proven [24] that for large $\lambda$

$$
\log H_{\infty}(\lambda) \sim \lambda^{\alpha} L\left(\frac{\log \lambda}{\log \langle k\rangle}\right),
$$

with $\alpha=\log p / \log \langle k\rangle$ as in (1.7) and $L(\cdot)$ a continuous positive periodic function of period 1. Harris was unable to establish that $L(\cdot)$ is not a constant as soon as $q_{p}<1$ (for $q_{p}=1$, that is for $F(x)=x^{p}$, it is straightforward to see that $L(\cdot)$ is constant) and this open issue has drawn the attention in the mathematical community (see for example [5, 6], dealing precisely with the problem left open by Harris) and similar - sometimes strictly related - oscillatory behaviors have been pointed out repeatedly (e.g. [27, 17, 33, 22, 37] and 8 for further references: the stress is often on the nearly constant and nearly sinusoidal character of these oscillations). To our knowledge, establishing in general that $L(\cdot)$ is non constant is still an open problem for $p>2$ (for $p=2$ the non triviality of $L(\cdot)$ is established in [8] by exploiting results in [10]).

Here again one can try to perturb around a situation where the map becomes simple. However there is the following important preliminary issue: what is the relation, if any, between the periodic functions $B(\cdot)$ and $L(\cdot)$, both of period one, appearing in (2.7) and in (2.16). A priori, these two periodic function come out of different questions that have in common only the underlying polynomial $F$. But in fact if the polynomial is the same in the two questions, then the periodic functions coincide up to the change of variable $B(y)=L(-y)$ (this has been proven in [8] and the need for switching the sign is due to the way we have defined $B(\cdot)$ and $L(\cdot)$ : the argument of proof is reproduced below). Given this observation, the analysis of $B(\cdot)$ and $L(\cdot)$ in the two perturbative limits we consider becomes just one problem.

2.3. Oscillations for hierarchical wetting and Galton-Watson models. We explain now how, by exploiting the two functional equations (1.5) and (2.14), one can see that the periodic function $B(\cdot)$ in (2.7) and $L(\cdot)$ in (2.16) coincide up to a sign change in the argument. For this we fix a polynomial $F(x)=\sum_{i=0}^{p} q_{i} x^{i}$, like in Remark 2.1 for the wetting model and in Remark 2.2 for the Galton-Watson process, and we define for every $\lambda>0$

$$
M(\lambda)=\lambda^{-\alpha} \psi_{\infty}\left(H_{\infty}(\lambda)\right),
$$

and by applying first (2.14) together with $\alpha=\log p / \log \langle k\rangle$, and then (1.5) we have

$$
M(\lambda\langle k\rangle)=\frac{\lambda^{-\alpha}}{p} \psi_{\infty}\left(F\left(H_{\infty}(\lambda)\right)\right)=\lambda^{-\alpha} \psi_{\infty}\left(H_{\infty}(\lambda)\right)=M(\lambda),
$$

that is, $M(\cdot)$ is multiplicatively periodic of period $\langle k\rangle$. Moreover $M(\cdot)$ is analytic on $(0, \infty)$ because $H_{\infty}(\cdot)$ is entire (cf. Remark 2.2) and $\psi_{\infty}(\cdot)$ is analytic on $(1, \infty)$ (cf. Remark 2.1). The fact that $M(\cdot)>0$ on $(0, \infty)$ is just the strict positivity of $\psi_{\infty}(x)$ for $x>1$ and the fact that $H_{\infty}(\lambda)>1$ for $\lambda>0$.

Now from the definitions it is immediate to see that $\psi_{\infty}(x) \sim \log x$ for $x \rightarrow \infty$ and that $\lim _{\lambda \rightarrow \infty} H_{\infty}(\lambda)=\infty$, so (2.17)

$$
\log \left(H_{\infty}(\lambda)\right) \stackrel{\lambda \rightarrow \infty}{\sim} \lambda^{\alpha} M(\lambda),
$$

which provides a proof of (2.16) and relates explicitly $M(\cdot)$ and $L(\cdot)$.

On the other hand (2.17) can be rewritten as

$$
\psi_{\infty}(x)=\left(H_{\infty}^{-1}(x)\right)^{\alpha} M\left(H_{\infty}^{-1}(x)\right) .
$$


But since $H_{\infty}(0)=1$ and $H_{\infty}^{\prime}(0)=1$, we see that $H_{\infty}^{-1}(1+y) \sim y$ for $y \searrow 0$, so that from (2.20) one directly obtains for $x \searrow 1$

$$
\psi_{\infty}(x) \sim(x-1)^{\alpha} M(x-1),
$$

which proves (2.7) and explicitly relates $B(\cdot)$ to $M(\cdot)$, and we see that $B(y)=L(-y)$ for every $y$. This completes the proof of the equivalence of the two example models we have proposed, as far as oscillations are concerned.

Remark 2.3. As it was pointed out in [20], the partition function of the hierarchical wetting model has a Galton-Watson representation:

$$
\frac{Z_{n}(T)}{Z_{n}(\infty)}=\left\langle\exp \left(\frac{a}{T} N_{n}\right)\right\rangle
$$

Formula (2.22) shows that the partition function of the hierarchical wetting model can be interpreted as the generating function of the size $N_{n}$ of the population at the $n$-th generation of a Galton-Watson processes. It also provides an expression for the partition function of the hierarchical model in terms of sum of Boltzmann weights when $b$ is not integer.

\section{MAIN RESUlts}

3.1. Oscillations for the map (1.1-1.9) for $\epsilon$ small. Here instead of writing the results in terms of the difference $x-x_{*}$, we found slightly more convenient to use $\log \left(x / x_{*}\right)$. One can of course make a simple change of variables to rewrite the final result in terms of the difference $x-x_{*}$. Then (1.6) becomes for $x$ close to $x_{*}$

$$
\psi_{\infty}(x) \simeq\left(\log \left[x / x_{*}\right]\right)^{\alpha} B\left(-\frac{\log \left(\log \left[x / x_{*}\right]\right)}{\log F^{\prime}\left(x_{*}\right)}\right),
$$

where $B(\cdot)$ is a periodic function. By replacing $\psi_{\infty}(x)$ by this leading behavior in (1.5) one can calculate subdominant contributions when $x \rightarrow x_{*}$ and one gets at next order

$$
\begin{aligned}
\psi_{\infty}(x) \simeq & \left(\log \left[x / x_{*}\right]\right)^{\alpha} B\left(-\frac{\log \left(\log \left[x / x_{*}\right]\right)}{\log F^{\prime}\left(x_{*}\right)}\right) \\
& -\left(\log \left[x / x_{*}\right]\right)^{\alpha+1} \frac{\alpha\left[x_{*} F^{\prime \prime}\left(x_{*}\right)+F^{\prime}\left(x_{*}\right)-F^{\prime}\left(x_{*}\right)^{2}\right]}{2 F^{\prime}\left(x_{*}\right)\left(F^{\prime}\left(x_{*}\right)-1\right)} \\
& \times\left[B\left(-\frac{\log \left(\log \left[x / x_{*}\right]\right)}{\log F^{\prime}\left(x_{*}\right)}\right)-\frac{1}{\log p} B^{\prime}\left(-\frac{\log \left(\log \left[x / x_{*}\right]\right)}{\log F^{\prime}\left(x_{*}\right)}\right)\right]+\ldots
\end{aligned}
$$

In fact all the subdominant contributions can be expressed in terms of derivatives of the map $F(x)$ evaluated at $x_{*}$ and of derivatives of the periodic function $B(y)$. So the problem of calculating the amplitudes of the dominant contribution and of all subdominant contributions reduces to the calculation of the periodic function $B(y)$.

One of our main results, derived below under the condition (1.9) is that

$$
B(y)=1+\epsilon B_{1}(y)+\epsilon^{2} B_{2}(y)+\ldots
$$

with

$$
B_{1}(y)=\frac{1}{p}\left[-y G^{\prime}(1)+\sum_{k=0}^{\infty} p^{y-k} G\left(e^{p^{k-y}}\right)+\sum_{k=-\infty}^{-1}\left[p^{y-k} G\left(e^{p^{k-y}}\right)-G^{\prime}(1)\right]\right],
$$


and

$$
\begin{aligned}
& B_{2}(y)=\frac{y(y+1)}{2 p^{2}} G^{\prime}(1)^{2} \\
&+\frac{1}{p}\left[\sum_{k \geq 0}\left(-\frac{p^{y-k}}{2} G^{2}\left(e^{p^{k-y}}\right)+e^{p^{k-y}} G^{\prime}\left(e^{p^{k-y}}\right)\left[B_{1}(y)-p^{y-k} U\left(e^{p^{k-y}}\right)\right]\right)\right. \\
&+\sum_{k=-\infty}^{-1}\left(-\frac{G^{\prime}(1)^{2}}{p}(k-y)-\frac{p^{y-k}}{2} G^{2}\left(e^{p^{k-y}}\right)\right. \\
&\left.\left.\quad+e^{p^{k-y}} G^{\prime}\left(e^{p^{k-y}}\right)\left[B_{1}(y)-p^{y-k} U\left(e^{p^{k-y}}\right)\right]\right)\right],
\end{aligned}
$$

where $U(x)$ is defined by

$$
U(x)=\frac{1}{p} \sum_{k=0}^{\infty} p^{-k} G\left(x^{p^{k}}\right) .
$$

Remark 3.1. In the particular case

$$
F(x)=(1-\epsilon) x^{p}+\epsilon
$$

one has $G(x)=x^{-p}-1$ and therefore

$$
B_{1}(y)=y+\sum_{k=1}^{\infty} g(y-k)+\sum_{k=-\infty}^{0}(g(y-k)+1),
$$

with

$$
g(y)=p^{y}\left(\exp \left(-p^{-y}\right)-1\right) .
$$

From this expression one can obtain the Fourier coefficients $a_{n}=\int_{0}^{1} \exp (-2 \pi n i y) B_{1}(y) \mathrm{d} y$ of the periodic function $B_{1}(\cdot)$ and one finds

$$
a_{0}=\frac{1}{2}-\frac{1+\Gamma^{\prime}(1)}{\log p}=\frac{1}{2}-\frac{1-\gamma}{\log p}
$$

$(\gamma=0.5772 \ldots$ is the Euler-Mascheroni constant) and for $n \neq 0$

$$
a_{n}=\frac{1}{\log p} \Gamma\left(\frac{2 i \pi n}{\log p}-1\right) .
$$

These espressions are compared in Table 1 with the Fourier coefficients calculated directly for a finite value of $\epsilon$.

Remark 3.2. (3.10) and (3.11) follow from (3.8) by direct computation. It is however worthwhile to point out that there is a direct link with a Poisson summation formula (and, for example, with a similar computation in a different context [35, § 3.3]). In fact, we have

$$
B_{1}^{\prime}(y)=1+\sum_{k=-\infty}^{\infty} g^{\prime}(y+k)
$$




\begin{tabular}{|l||l|l|l|l|l|}
\hline $1 / \epsilon$ & $\left(a_{0}-1\right) / \epsilon$ & $\Re\left(a_{1} / \epsilon\right) \times 10^{8}$ & $\Im\left(a_{1} / \epsilon\right) \times 10^{8}$ & $\Re\left(a_{2} / \epsilon\right) \times 10^{14}$ & $\Im\left(a_{2} / \epsilon\right) \times 10^{14}$ \\
\hline \hline 10 & 0.129 & -3.65 & -7.08 & 1.47 & -0.80 \\
$10^{2}$ & -0.093 & -4.80 & -7.07 & 1.62 & -1.11 \\
$10^{3}$ & -0.108 & -4.788 & -7.164 & 1.682 & -1.085 \\
$10^{4}$ & -0.0978 & -4.786 & -7.174 & 1.6887 & -1.0819 \\
$10^{5}$ & -0.10993 & -4.7858 & -7.1753 & 1.68938 & -1.08166 \\
\hline$\infty$ & -0.10995 & -4.7858 & -7.1757 & 1.689459 & -1.081618 \\
\hline
\end{tabular}

TABLE 1. For $F(x)=(1-\epsilon) x^{2}+\epsilon$ the limit $\epsilon \searrow 0$ expressions in (3.10) and in (3.11) are compared with values for $\epsilon>0$ obtained by using formula (2.17): an accurate expression for $H_{\infty}(\cdot)$ is obtained by writing enough terms in the Taylor expansion at zero (these terms are easily obtained recursively by using $H_{\infty}(0)=1, H_{\infty}^{\prime}(0)=1$ and by taking derivatives of the Poincaré equation (2.14) ) and by exploiting the series in (2.9) to get a good approximation of $\psi_{\infty}(\cdot)$.

so the $n$-th Fourier coefficient of $B_{1}^{\prime}(\cdot)$ is equal to $\int_{-\infty}^{\infty} g^{\prime}(y) \exp (-2 \pi i n y) \mathrm{d} y=: \widehat{g^{\prime}}(2 \pi n)$ for $n \neq 0$ (Poisson summation formula, see e.g. [34, Ch. 4]), while the 0-th Fourier coefficient is of course 0 . It is then straightforward to see that $a_{n}=i \widehat{g^{\prime}}(2 \pi n) / 2 \pi n$ and (3.11) can be recovered this way too.

3.2. Large $p$ limit of the map (1.11). For the map (1.11), for $q$ and $P(\cdot)$ fixed and $p$ large, so that in particular $F^{\prime}(1)=q p+(1-q) P^{\prime}(1) \sim q p$, our result is that if

$$
-\frac{\log (x-1)}{\log \left(F^{\prime}(1)\right)}=n(x)+y \quad \text { with } \quad \eta<y<\eta+1,
$$

where $n(x)$ is an integer, then for $y \neq 1$ as $p \rightarrow \infty$

$$
\psi_{\infty}(x) \simeq \begin{cases}(x-1)^{\alpha} q^{-y} & \text { for } \eta<y<1, \\ (x-1)^{\alpha} q^{1-y} & \text { for } 1<y<1+\eta,\end{cases}
$$

This gives a discontinuous amplitude at $y=1$. Recalling (1.6), an equivalent way to state (3.14) is to say that for every $y \notin \mathbb{Z}$

$$
\lim _{p \rightarrow \infty} B(y)=q^{-\{y\}},
$$

where $\{x\}$ is the fractionary part of $x$, that is $\{x\}=x-\max \{n \in \mathbb{Z}: n \leq x\}$.

One can resolve the discontinuity at $y=0$ by analyzing, in the large $p$ limit, the range $y-1 \sim(\log p)^{-1}$ and one gets

$$
\psi_{\infty}(x) \simeq(x-1)^{\alpha} e^{-(1-y) \log p} \log \left[q \exp \left(\frac{e^{(1-y) \log p}}{q}\right)+1-q\right]
$$

Note also that in the case $q=1-\epsilon$ and $P(\cdot) \equiv 1$ for large $p$ one gets from (3.4)-(3.5) in the range $0<y<1$

$$
B_{1}(y)=y \quad \text { and } \quad B_{2}(y)=\frac{y(y+1)}{2},
$$

which agrees with (3.14) when one takes the first two terms in the expansion in powers of $\epsilon$. 


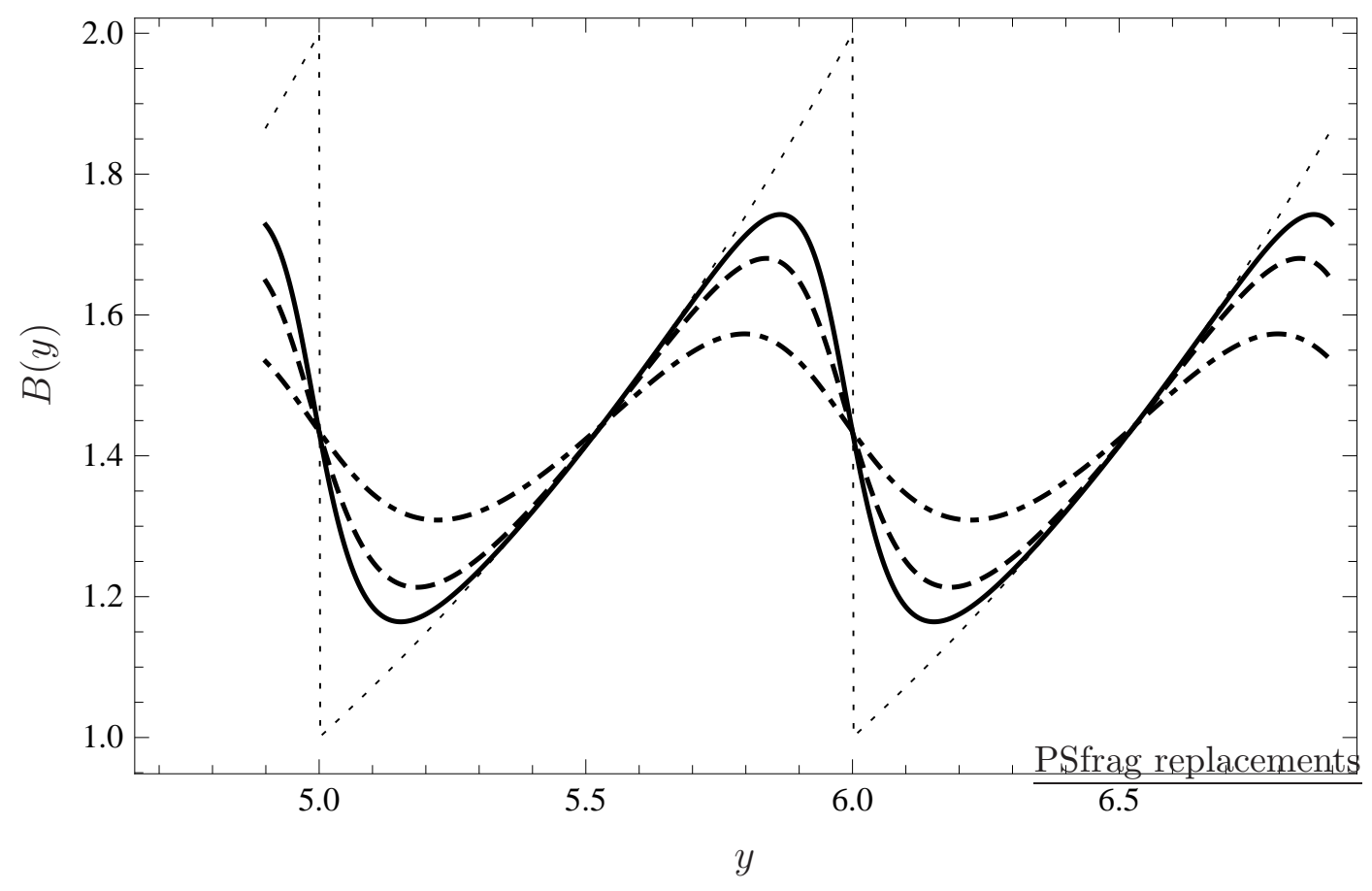

FiguRE 1. With the choice $F(x)=\left(x^{p}+1\right) / 2$ we have plotted $B(\cdot)$ for $p=10^{3}$ (dot-dashed line), $10^{5}$ (dashed line) and $10^{7}$ (solid line), and the this dotted line for $p=\infty$. For the $p=\infty$ case we have exploited the result (3.15), that is we have plotted $y \mapsto 2^{\{y\}}$. The numerical data for $p<\infty$ have been obtained exploiting (2.9). The convergence is rather slow simply because the effective large parameter is $\log p$. Notice that already at $p=1000 B(\cdot)$ is far from being nearly constant and starting from $p=10^{5}$ it is also evident that it is not nearly sinusoidal. These large amplitudes contrast with the small amplitudes one usually observes for small $p$ [5, 6, 13, 8].

\section{Perturbation in powers of $\epsilon$ FAR From the CRitical Point}

Our approach consists first in calculating in this section $\psi_{\infty}(x)$ in powers of $\epsilon$, for $x \neq x_{*}$. Then by matching (in Section 6) the singularities (obtained in Section 5) of the successive terms of the expansion with the expected expansion (see Section 6) near the critical point, one gets the periodic functions $B_{1}$ and $B_{2}$.

Starting from the map (1.1) and defining the successive terms of the expansion of $F^{(n)}(x)$ in powers of $\epsilon$ by

$$
F^{(n)}(x)=x^{p^{n}} \exp \left(\epsilon p^{n} Y_{n}(x)+\epsilon^{2} p^{n} Z_{n}(x)+O\left(\epsilon^{3}\right)\right) .
$$

Then by replacing in the expression (1.1) one gets

$$
\begin{aligned}
& Y_{n}(x)=Y_{n-1}(x)+p^{-n} G\left(x^{p^{n-1}}\right), \\
& Z_{n}(x)=Z_{n-1}(x)-\frac{1}{2 p^{n}} G^{2}\left(x^{p^{n-1}}\right)+\frac{1}{p} G^{\prime}\left(x^{p^{n-1}}\right) x^{p^{n-1}} Y_{n-1}(x) .
\end{aligned}
$$


One can show by recursion that

$$
Y_{n}(x)=\frac{1}{p} \sum_{k=0}^{n-1} p^{-k} G\left(x^{p^{k}}\right)=U(x)-\frac{U\left(x^{p^{n}}\right)}{p^{n}},
$$

where we recall $U(x)$ from (3.6)

$$
U(x)=\frac{1}{p} \sum_{k=0}^{\infty} p^{-k} G\left(x^{p^{k}}\right),
$$

and this leads to

$$
\begin{aligned}
\psi_{n}(x)= & \log x+\epsilon Y_{n}(x)+\epsilon^{2} Z_{n}(x)+O\left(\epsilon^{3}\right) \\
= & \log x+\epsilon\left(U(x)-\frac{U\left(x^{p^{n}}\right)}{p^{n}}\right)-\frac{\epsilon^{2}}{2 p} \sum_{k=0}^{n-1} p^{-k} G\left(x^{p^{k}}\right)^{2} \\
& +\frac{\epsilon^{2}}{p} \sum_{k=0}^{n-1} x^{p^{k}} G^{\prime}\left(x^{p^{k}}\right)\left(U(x)-\frac{U\left(x^{p^{k}}\right)}{p^{k}}\right)+O\left(\epsilon^{3}\right) .
\end{aligned}
$$

For large $n$, assuming $G(\cdot)$ is such that the sums converge, one gets

$$
\psi_{\infty}(x)=\log x+\epsilon U(x)+\epsilon^{2}\left(-\frac{V_{1}(x)}{2}+U(x) V_{2}(x)-V_{3}(x)\right)+\ldots
$$

where

$$
\begin{aligned}
& V_{1}(x)=\frac{1}{p} \sum_{k=0}^{\infty} p^{-k} G^{2}\left(x^{p^{k}}\right), \\
& V_{2}(x)=\frac{1}{p} \sum_{k=0}^{\infty} x^{p^{k}} G^{\prime}\left(x^{p^{k}}\right), \\
& V_{3}(x)=\frac{1}{p} \sum_{k=0}^{\infty} p^{-k} x^{p^{k}} G^{\prime}\left(x^{p^{k}}\right) U\left(x^{p^{k}}\right) .
\end{aligned}
$$

Since $G(\cdot)$ is bounded, the first series converges. Moreover if the series for $V_{2}(\cdot)$ converges, so does the series defining $V_{3}(\cdot)$. Therefore we require the converges of the series defining $V_{2}(\cdot)$ and this amounts to an additional assumption on the large $x$ behavior of $G^{\prime}(x)$ : for example, it suffices that $\left.\left|G^{\prime}(x)\right| \leq 1 / x(\log x)^{a}\right)$ for some $a>1$ and $x$ large.

\section{Singular Behavior of the Sums (3.6)-(4.7)}

In this section we try to extract the singular behavior, as $x \searrow 1$, of the sums $U(x), V_{1}(x), V_{2}(x)$ and $V_{3}(x)$ defined in (3.6)-(4.7).

For $x$ sufficiently close to 1 one can write

$$
\log (\log x)=-\left(n_{0}+y\right) \log p
$$

$n_{0}$ is an integer where $0<y<1$. Then as $x \searrow 1$, the integer $n_{0} \rightarrow \infty$.

We will also assume that as in (1.9), one has $G(1)=0$, and since $G(\cdot)$ is smooth

$$
G(x)=G^{\prime}(1)(x-1)+G^{\prime \prime}(1) \frac{(x-1)^{2}}{2}+\ldots
$$


5.1. Analysis of $U(x)$ as $x \searrow 1$. One can easily see, using $G(1)=0$ and the definition (5.1) of $n_{0}$, that $U(x)$ defined in (3.6) can be written as

$$
\begin{array}{r}
U(x)=\frac{\log x}{p}\left(\sum_{k=0}^{\infty} p^{y-k} G\left(e^{p^{k-y}}\right)+\sum_{k=-n_{0}}^{-1}\left[p^{y-k} G\left(e^{p^{k-y}}\right)-G^{\prime}(1)\right]\right) \\
+\frac{\log x}{p}\left(-\frac{\log (\log x)}{\log p}-y\right) G^{\prime}(1),
\end{array}
$$

Then, by using (5.2), we see that $n_{0} \rightarrow \infty$ as $x \searrow 1$ and

$$
U(x)-U^{*}(x)=O\left(\log ^{2} x\right),
$$

where $U^{*}(x)$ is defined as

$$
U^{*}(x)=-\frac{G^{\prime}(1)}{p \log p}(\log x)(\log (\log x))+B_{1}(y) \log x,
$$

where $B_{1}(\cdot)$ is the periodic function $\left(B_{1}(y)=B_{1}(y+1)\right)$ defined in (3.4).

A few remarks:

(1) One can show that

$$
\begin{gathered}
U(x)=\frac{1}{p} U\left(x^{p}\right)+\frac{1}{p} G(x), \\
U^{*}(x)=\frac{1}{p} U^{*}\left(x^{p}\right)+\frac{1}{p} G^{\prime}(1) \log x .
\end{gathered}
$$

(2) One can also evaluate the correction $U(x)-U^{*}(x)$ and get for $x \searrow 1$

$$
U(x)=U^{*}(x)-\frac{G^{\prime \prime}(1)+G^{\prime}(1)}{2(p-1) p} \log ^{2}(x)+O\left(\log ^{3}(x)\right) .
$$

(3) One can check directly from the full expression (3.4) of $B_{1}(y)$ that

$$
B_{1}(y+1)=B_{1}(y) .
$$

5.2. Analysis of $V_{1}(x)$ as $x \searrow 1$. Using the definition (5.1) of $n_{0}$ and the behavior (5.2) of $G(x)$ when $x \searrow 1$ one can rewrite the sum in the first line of (4.7) as

$$
V_{1}(x)(x)=\frac{\log x}{p} \sum_{k=-n_{0}}^{\infty} p^{y-k} G^{2}\left(e^{p^{k-y}}\right),
$$

and as $n_{0} \rightarrow \infty$ in the limit $x \searrow 1$ one gets

$$
V_{1}(x) \simeq C_{1}(y) \log x,
$$

where $C_{1}(\cdot)$ is the periodic function $\left(C_{1}(y+1)=C_{1}(y)\right)$ defined by

$$
C_{1}(y)=\frac{1}{p} \sum_{k=-\infty}^{\infty} p^{y-k} G^{2}\left(e^{p^{k-y}}\right) .
$$


5.3. Analysis of $V_{2}(x)$ as $x \searrow 1$. Using again the relation (5.1) between $x$ and $n_{0}$ one can write the sum in the second expression in (4.7)

$$
\begin{gathered}
V_{2}(x)=\frac{1}{p}\left[\sum_{k=0}^{\infty} e^{p^{k-y}} G^{\prime}\left(e^{p^{k-y}}\right)+\sum_{k=-n_{0}}^{-1} e^{p^{k-y}} G^{\prime}\left(e^{p^{k-y}}\right)-G^{\prime}(1)\right]+\frac{G^{\prime}(1)}{p} n_{0} \simeq \\
\frac{1}{p}\left[\sum_{k=0}^{\infty} e^{p^{k-y}} G^{\prime}\left(e^{p^{k-y}}\right)+\sum_{k=-\infty}^{-1}\left[e^{p^{k-y}} G^{\prime}\left(e^{p^{k-y}}\right)-G^{\prime}(1)\right]\right]-\frac{\log (\log x)}{p \log p} G^{\prime}(1)-\frac{G^{\prime}(1)}{p} y .
\end{gathered}
$$

One then finds

$$
V_{2}(x)=-G^{\prime}(1) \frac{\log (\log x)}{p \log p}+C_{2}(y)+O(\log x),
$$

with with the periodic function $C_{2}(\cdot)$ given by

$$
C_{2}(y)=\frac{1}{p}\left[-y G^{\prime}(1)+\sum_{k=0}^{\infty} e^{p^{k-y}} G^{\prime}\left(e^{p^{k-y}}\right)+\sum_{k=-\infty}^{-1}\left[e^{p^{k-y}} G^{\prime}\left(e^{p^{k-y}}\right)-G^{\prime}(1)\right]\right] .
$$

Remark 5.1. One can easily see from the definitions (3.6) and (4.7) of $U(x)$ and $V_{2}(x)$ that

$$
V_{2}(x)=x U^{\prime}(x)
$$

This implies that

$$
C_{2}(y)=B_{1}(y)-\frac{B_{1}^{\prime}(y)}{\log p}-\frac{G^{\prime}(1)}{p \log p},
$$

which can be also checked directly.

5.4. Analysis of $V_{3}(x)$ as $x \searrow 1$. From the definitions in the third expression in (4.7) and (5.1) of the sum $V_{3}$ and of $n_{0}$ one can write

$$
V_{3}(x)=\frac{\log x}{p} \sum_{k \geq 0} p^{-k+n_{0}+y} e^{p^{k-n_{0}-y}} G^{\prime}\left(e^{p^{k-n_{0}-y}}\right) U\left(e^{p^{k-n_{0}-y}}\right),
$$

which can be rewritten using again (5.1)

$$
\begin{aligned}
V_{3}(x)= & \frac{\log x}{p}\left[\sum_{k \geq 0} p^{-k+y} e^{p^{k-y}} G^{\prime}\left(e^{p^{k-y}}\right) U\left(e^{p^{k-y}}\right)\right. \\
& +\sum_{k \geq-n_{0}}^{-1}\left\{p^{-k+y} e^{p^{k-y}} G^{\prime}\left(e^{p^{k-y}}\right) U\left(e^{p^{k-y}}\right)+\frac{G^{\prime}(1)^{2}}{p}(k-y)-B_{1}(y) G^{\prime}(1)\right\} \\
& +\frac{G^{\prime}(1)^{2}}{2 p}\left(\frac{-\log (\log x)}{\log p}-y\right)\left(\frac{-\log (\log x)}{\log p}-y+1\right) \\
& \left.+\left(\frac{y G^{\prime}(1)^{2}}{p}+B_{1}(y) G^{\prime}(1)\right)\left(\frac{-\log (\log x)}{\log p}-y\right)\right] .
\end{aligned}
$$


Now from (5.5) one has for large negative $k$

$$
U\left(e^{p^{k-y}}\right)=p^{k-y}\left(-\frac{G^{\prime}(1)}{p}(k-y)+B_{1}(y)\right),
$$

so that one can take the $n_{0} \rightarrow \infty$ limit in the last expression of $V_{3}(x)$ to get

$$
\begin{aligned}
V_{3}(x)=-\frac{\log x \log (\log x)}{p \log p} & B_{1}(y) G^{\prime}(1) \\
& +\frac{G^{\prime}(1)^{2}}{2 p^{2}} \frac{\log x \log (\log x)}{\log p}\left(\frac{\log (\log x)}{\log p}-1\right)+C_{3}(y) \log x,
\end{aligned}
$$

where the periodic function $C_{3}(y)$ is defined by

$$
\begin{gathered}
C_{3}(y)=\frac{1}{p}\left[-y B_{1}(y) G^{\prime}(1)-y(y+1) \frac{G^{\prime}(1)^{2}}{2 p}+\sum_{k \geq 0} p^{-k+y} e^{p^{k-y}} G^{\prime}\left(e^{p^{k-y}}\right) U\left(e^{p^{k-y}}\right)\right. \\
\left.+\sum_{k=-\infty}^{-1}\left\{p^{-k+y} e^{p^{k-y}} G^{\prime}\left(e^{p^{k-y}}\right) U\left(e^{p^{k-y}}\right)+\frac{G^{\prime}(1)^{2}}{p}(k-y)-B_{1}(y) G^{\prime}(1)\right\}\right] .
\end{gathered}
$$

Here again one can check directly in(5.22) that $C_{3}(y+1)=C_{3}(y)$.

\section{Expected EXPRESSION OF $\psi_{\infty}(\cdot)$}

Since here $G(1)=0$ and $x_{*}=1$, the expected critical behavior (3.2) of $\psi_{\infty}(\cdot)$ should be of the form

$$
\psi_{\infty}(x) \simeq(\log x)^{\alpha} B\left(-\frac{\log (\log x)}{\log F^{\prime}(1)}\right)+O\left((\log x)^{\alpha+1}\right) .
$$

For $\epsilon$ small one has

$$
F^{\prime}(1)=p+\epsilon f_{1}, \quad \alpha=1+\epsilon a_{1}+\epsilon^{2} a_{2}+\ldots \quad \text { and } B(y)=1+\epsilon B_{1}(y)+\epsilon^{2} B_{2}(y)+\ldots
$$

with

$$
f_{1}=G^{\prime}(1), \quad a_{1}=-\frac{G^{\prime}(1)}{p \log p} \text { and } a_{2}=\frac{G^{\prime}(1)^{2}(2+\log p)}{2(p \log p)^{2}}
$$

and one gets from (6.1)

$$
\begin{aligned}
\psi_{\infty}(x)= & \left.\log x+\left[B_{1}(y) \log x+a_{1} \log x \log (\log x)\right)\right] \epsilon \\
+\left[B_{2}(y) \log x\right. & +a_{2} \log (x) \log (\log (x))+a_{1} B_{1}(y) \log x \log (\log (x)) \\
& \left.+\frac{a_{1}^{2}}{2} \log x[\log (\log x)]^{2}+f_{1} \frac{\log x \log (\log x)}{p(\log p)^{2}} B_{1}^{\prime}(y)\right] \epsilon^{2}+\ldots
\end{aligned}
$$

where

$$
y=-\frac{\log (\log x)}{\log p} .
$$

Comparing with (4.6) and (6.4) with $U(x), V_{1}(x), V_{2}(x)$ and $V_{3}(x)$ replaced by their estimates (5.5), (5.11), (5.14) and (5.21) one then finds

$$
B_{2}(y)=-\frac{C_{1}(y)}{2}+B_{1}(y) C_{2}(y)-C_{3}(y)
$$


which leads to (3.5) once the periodic functions $C_{1}, C_{2}, C_{3}$ have been replaced by the expressions (5.12), (5.15) and (5.22).

\section{LARGE $p$ LIMIT}

We consider now the the map (1.11): recall that $q \in(0,1)$ is fixed, as well as the polynomial $P(\cdot)$. When $p$ is large $x_{*}=1, F^{\prime}(1)=p q+(1-q) P^{\prime}(1) \sim p q$ and one has (compare with (1.7))

$$
\alpha=\frac{\log p}{\log F^{\prime}(1)} \simeq 1-\frac{\log q}{\log p} .
$$

For $x$ close to 1 , let us define $z$ by

$$
1+\left(F^{\prime}(1)\right)^{n(x)}(x-1)=1+\frac{z}{p},
$$

where $n(x)$ is the integer such that

$$
p^{-\eta} \leq z<F^{\prime}(1) p^{-\eta} \stackrel{p \rightarrow \infty}{\sim} q p^{1-\eta},
$$

and $\eta \in(0,1)$ is some fixed number (for example $\eta=1 / 3$ ).

The main idea is that for $x$ close to 1 , the map (1.11) can be replaced by $F(x) \simeq$ $1+F^{\prime}(1)(x-1)$ for the first $n(x)$ iterations, and by $F(x) \simeq q x^{p}$ beyond the $n(x)+2^{\text {nd }}$ iteration. Only the $n(x)+1^{\text {st }}$ iterate has to be calculated with the full expression (1.11).

Therefore recalling that $P(1)=1$ we have

$$
F^{n(x)+m+1}(x) \simeq\left(q e^{z}+1-q\right)^{p^{m}} q^{\frac{p^{m}-1}{p-1}},
$$

and

$$
\frac{\log F^{n(x)+m+1}(x)}{p^{n(x)+1+m}}=\frac{1}{p} p^{-n(x)}\left[\log \left(q e^{z}+1-q\right)+\left(\frac{1}{p-1}-\frac{1}{p^{m}(p-1)}\right) \log q\right],
$$

and by taking the $m \rightarrow \infty$ limit and using $\left(F^{\prime}(1)\right)^{\alpha}=p$ we obtain

$$
\psi_{\infty}(x) \simeq \frac{(x-1)^{\alpha}}{p}\left(\frac{p}{z}\right)^{\alpha}\left[\log \left(q e^{z}+1-q\right)+\frac{\log q}{p-1}\right] .
$$

Taking the large $p$ limit we therefore obtain

$$
\psi_{\infty}(x) \simeq(x-1)^{\alpha} \frac{\log \left(q e^{z}+1-q\right)}{q z^{\alpha}} .
$$

One can then write

$$
-\frac{\log (x-1)}{\log F^{\prime}(1)}=n(x)+y \quad \text { with } \quad \eta<y<\eta+1,
$$

that is

$$
n(x)=\left\lfloor-\frac{\log (x-1)}{\log F^{\prime}(1)}-\eta\right\rfloor,
$$

where $\lfloor a\rfloor:=\max \{n \in \mathbb{Z}: n \leq a\}$, or equivalently (see (17.2) )

$$
\frac{z}{p}=\left(F^{\prime}(1)\right)^{-y}
$$

and note that in the large $p$ limit $z \nearrow \infty$ (respectively $z \searrow 0$ ) for $y<1$ (respectively $y>1)$. Then one finds, for fixed $y \neq 1$

$$
\psi_{\infty}(x) \simeq \begin{cases}(x-1)^{\alpha} q^{-y} & \text { for } \eta \leq y<1, \\ (x-1)^{\alpha} q^{1-y} & \text { for } 1<y<1+\eta,\end{cases}
$$


as claimed in (3.14).

At $y=1$ the amplitude becomes discontinuous. One can resolve this discontinuity by analyzing, in the large $p$ limit, the range $y-1 \sim(\log p)^{-1}$ and one gets (3.16) from (7.1) and (7.7).

\section{ACKNOWLEDGEMENTS}

The authors acknowledge the support of ANR, grant SHEPI.

\section{REFERENCES}

[1] E. Akkermans, O. Benichou, G. V. Dunne, A. Teplyaev and R. Voituriez, Spatial log periodic oscillations of first-passage observables in fractals, arXiv:1207.3298

[2] E. Akkermans, G. V. Dunne and A. Teplyaev, Physical consequences of complex dimensions of fractals, Eur. Phys. Lett. 88 (2009), 40007.

[3] A. F. Beardon, Iteration of rational functions, Graduate Texts in Mathematics, 132, Springer-Verlag, New York, 1991.

[4] A. N. Berker and S. Ostlund, Renormalisation-group calculations of finite systems: order parameter and specific heat for epitaxial ordering, J. Phys. C 12 (1979), 4961-4975.

[5] J. D. Biggins and N. H. Bingham, Near-constancy phenomena in branching processes, Math. Proc. Cambridge Phil. Soc. 110 (1991), 545-558.

[6] J. D. Biggins and S. Nadarajah, Near-constancy of the Harris function in the simple branching process, Communications in Statistics. Stochastic Models 9 (1993), 435-444.

[7] C. de Calan, J. M. Luck, T. M. Nieuwenhuizen and D. Petritis, On the distribution of a random variable occurring in 1d disordered-systems, J. Phys. A: Math. Gen. 18 (1985), 501-523.

[8] O. Costin and G. Giacomin, Oscillatory critical amplitudes in hierarchical models and the Harris function of branching processes, J. Statist. Phys. 150 (2013), 471-486.

[9] O. Costin and M. Huang, Behavior of lacunary series at the natural boundary, Adv. Math. 222 (2009), 1370-1404.

[10] O. Costin and M. Huang, Geometric construction and analytic representation of Julia sets of polynomial maps, Nonlinearity 24 (2011), 1311-1327.

[11] B. Derrida, V. Hakim and J. Vannimenus, Effect of disorder on two-dimensional wetting, J. Stat. Phys. 66 (1992), 1189-1213.

[12] B. Derrida, H. J. Hilhorst, Singular behavior of certain infinite products of random 2 X 2 matrices, J. Phys. A16 (1983), 2641-2654.

[13] B. Derrida, C. Itzykson and J. M. Luck, Oscillatory critical amplitudes in hierarchical models, Commun. Math. Phys. 94 (1984), 115-132.

[14] B. Derrida, S. C. Manrubia and D. H. Zanette, Distribution of repetitions of ancestors in genealogical trees Physica A 281 (2000), 1-16.

[15] B. Derrida, L. De Seze and C. Itzykson, Fractal structure of zeros in hierarchical models, J. Statist. Phys. 33 (1983), 559-569.

[16] B. Douçot, W. Wang, J. Chaussy, B. Pannetier, R. Rammal, A. Vareille and D. Henry, First observation of the universal periodic corrections to scaling: Magnetoresistance of normal-metal self-similar networks Phys. Rev. Lett. 57 (1986), 1235-1238.

[17] S. Dubuc, Etude théorique et numérique de la fonction de Karlin-McGregor, J. Analyse Math. 42 (1982), 15-37.

[18] G. V. Dunne, Heat kernels and zeta functions on fractals, J. Phys. A: Math. Theor. 45 (2012), 374016.

[19] G. Giacomin, H. Lacoin and F. L. Toninelli, Hierarchical pinning models, quadratic maps and quenched disorder, Probab. Theory Relat. Fields 147 (2010), 185-216.

[20] G. Giacomin, H. Lacoin and F. L. Toninelli, Marginal relevance of disorder for pinning models, Comm. Pure Appl. Math. 63 (2010), 233-265.

[21] S. Gluzman and D. Sornette, Log-periodic route to fractal functions Phys. Rev. E 65 (2002), 036142.

[22] P. J. Grabner and W. Woess, Functional iterations and periodic oscillations for simple random walk on the Sierpinski graph, Stochastic Process. Appl. 69 (1997), 127-138.

[23] R. B. Griffiths and M. Kaufman, Spin systems on hierarchical lattices. Introduction and thermodynamic limit Phys. Rev. B 26 (1982), 5022-5032.

[24] T. E. Harris, Branching processes, Ann. Math. Statist. 41 (1948), 474-494. 
[25] T. E. Harris, The Theory of Branching Processes, Springer-Verlag, 1963.

[26] D. Karevski and L. Turban, Log-periodic corrections to scaling: Exact results for aperiodic Ising quantum chains J. Phys. A: Math. Gen. 29 (1996), 3461-3470.

[27] S. Karlin and J. McGregor, Embeddability of discrete-time branching processes into continuous-time branching processes, Trans. Amer. Math. Soc. 132 (1968), 115-136.

[28] M. Kaufman and R. B. Griffiths, Exactly soluble Ising models on hierarchical lattices, Phys. Rev. B 24 (1981), 496-498.

[29] J. C. Lessa and R. F. S. Andrade, Log-periodic oscillations for a uniform spin model on a fractal Phys. Rev. E 62 (2000), 3083-3089.

[30] J. Milnor, Dynamics in one complex variable, third edition, Annals of Mathematics Studies 160, Princeton University Press, 2006.

[31] C. Monthus and T. Garel, Critical behavior of interfaces in disordered Potts ferromagnets: Statistics of free-energy, energy, and interfacial adsorption Phys. Rev. B 77 (2008), 134416.

[32] F. A. B. F. de Moura, U. Tirnakli and M. L. Lyra, Convergence to the critical attractor of dissipative maps: Log-periodic oscillations, fractality, and nonextensivity Phys. Rev. E 62 ( 2000), 6361-6365.

[33] A. M. Odlyzko, Periodic oscillations of coefficients of power series that satisfy functional equations, Adv. in Math. 44 (1982), 180-205.

[34] M. A. Pinsky, Introduction to Fourier analysis and wavelets, Graduate Studies in Mathematics 102, American Mathematical Society, 2009.

[35] D. Sornette, Discrete-scale invariance and complex dimensions, Physics Reports, 297 (1998), 239-270.

[36] D. Stauffer and D. Sornette, Log-periodic oscillations for biased diffusion on random lattice, Physica A 252 (1998), 271-277.

[37] E. Teufl, On the asymptotic behaviour of analytic solutions of linear iterative functional equations, Aequationes Math. 73 (2007), 18-55.

Laboratoire de Physique Statistique, École Normale Supérieure, Université Pierre et Marie Curie, Université Paris Diderot, CNRS, 24, Rue Lhomond, 75231 Paris Cedex 05 FRANCE

Université Paris Diderot and Laboratoire de Probabilités et Modèles Aléatoires, U.F.R. Mathématiques, BÂt. Sophie Germain, 5 rue Thomas Mann, 75205 PARIS Cedex 13 - France 